\title{
IMPACTO DE LA EMERGENCIA SANITARIA COVID-19 EN LA DECLARACIÓN DE IMPUESTOS DE LOS COMERCIANTES DEL CANTÓN PEDRO CARBO
}

\author{
AUTORES: Ricardo Junior Merchan Holguin ${ }^{1}$ \\ Adela del Jesús Lucio Pillasagua ${ }^{2}$
}

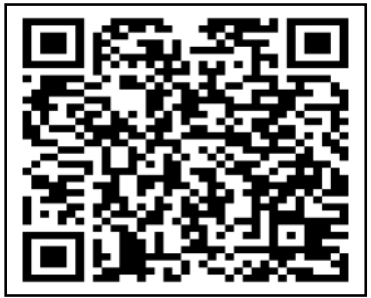

DIRECCIÓN PARA CORRESPONDENCIA: merchan-ricardo1565@unesum.edu.ec

Fecha de recepción: 12/07/2021

Fecha de aceptación: 10/01/2022

\section{RESUMEN}

Las declaraciones de impuestos son parte fundamental para que la Administración Tributaria en la cual se reportan los ingresos percibidos por enajenación de bienes o servicios. El presente trabajo tiene como propósito analizar el impacto de la emergencia sanitaria Covid-19 en la declaración de impuestos de los comerciantes del cantón Pedro Carbo, el cual busca verificar el déficit de los valores tributarios percibidos del periodo 2020, los mercantes se acogieron a las disposiciones dadas por el COE Cantonal, para salvaguardar y mantener controlada la propagación del virus en el cual se vio afectado el ingresos económico, en el cantón Pedro Carbo se vio afectada la actividad comercial en donde el ingreso del consumidor se vio disminuida, Se efectuó metodología descriptiva donde la tabulación de datos es base importante para demostrar y conocer el impacto en la baja de recaudaciones de impuestos, bibliográfico que a través revistas científicas, libros, informes de titulación y normativas vigentes aportando a fomentar esta investigación con bases e información legal, método inductivo permitió elaborar conclusiones de acuerdo las situaciones o sucesos que se hayan desarrollado, a los resultados encontrados se verifico $46 \%$ menos de ingresos tributarios, marzo con un $39 \%$ menos y septiembre con un $37 \%$ de déficit en ingresos de declaraciones que de manera general al año descendió de un $15 \%$ menos. En la cual se concluye que este déficit se debe a la emergencia sanitaria durante todo el periodo 2020 y las restricciones tomadas por los entes encargados de regular la emergencia.

PALABRAS CLAVE: Impacto; Declaración; Emergencia Sanitaria; Comerciantes; Impuestos.

\section{IMPACT OF THE COVID-19 HEALTH EMERGENCY ON THE TAX RETURN OF MERCHANTS IN THE PEDRO CARBO CANTON}

\footnotetext{
${ }^{1}$ Estudiante preprofesional de la carrera de Contabilidad y Auditoría en la Universidad Estatal del Sur de Manabí, cantón Paján - Ecuador. Orcid: https://orcid.org/0000-0001-9425-5036. E-mail: merchanricardo1565@unesum.edu.ec

${ }^{2}$ Docente de la carrera de Contabilidad y Auditoría de la Facultad de Ciencias Económicas, Universidad Estatal del Sur de Manabí, cantón Jipijapa- Ecuador. Orcid: http://orcid.org/0000-0001-6099-9168. E-mail: adela.lucio@unesum.edu.ec
} 
Ricardo Junior Merchan Holguin, Adela del Jesús Lucio Pillasagua

\section{ABSTRACT}

Taxes returns are a fundamental part of the Tax Administration in which the income received from the sale of properties or services is reported. The purpose of this work is to analyze the impact of the Covid-19 health emergency on the tax return of the merchants of Pedro Carbo town, which seeks to verify the deficit of the tax values received for the 2020 period, the merchants availed themselves of the provisions given by the COE local, to safeguard and control the spread of the virus in which it was affected the economic income, in the Pedro Carbo canton, the commercial activity was affected where the consumer's income was decreased, a descriptive methodology was carried out where the data tabulation is an important basis to demonstrate and know the impact on the decrease in tax collections, bibliographic that through scientific journals, books, degree reports and normat current taxes contributing to promote this research with bases and legal information, the inductive method allowed us to draw conclusions according to the situations or events that have developed, the results found were verified $46 \%$ less tax revenue, March with $39 \%$ less and September with a $37 \%$ deficit in income from declarations that in general per year fell from $15 \%$ les. In which it is concluded that this deficit is due to the health emergency throughout the 2020 period and the restrictions taken by the entities responsible for regulating the emergency.

KEYWORDS: Impact; Declaration; Health Emergency; Merchants; Taxes.

\section{INTRODUCCIÓN}

Para el Servicio de Rentas Internas, las declaraciones de impuestos de los contribuyentes son parte esencial para facultad recaudatoria de la administración tributaria, estos valores son declarados y pagados de manera periódica por la actividad económica a la cual se le atribuye el incremento de la base imponible tributaria, recolectada por el (SRI) entidad recaudadora en Ecuador, lo recaudado se redirige a el Presupuesto General del Estado donde se va administrar los recursos económicos del país.

Las declaraciones tributarias según el tipo de contribuyentes y su actividad deben de ser declarada en los periodos establecidos por la Administración tributaria en su normativa vigentes establecida según su tipología a las que se le adjunte sus obligaciones tributarias.

En Ecuador, los impuestos son un presupuesto que se recolecta por periodos según su tipo de gravamen, Impuesto a la Renta, Impuestos al Valor Agregado, Impuesto a la Salida de Divisas, Impuesto a los Consumos Especiales, Impuesto a los Vehículos Motorizados, Impuesto Redimible Botellas Plásticas no Retornables, Impuesto a los Activos en el Exterior los cuales disminuyeron alrededor de un $13 \%$ en porcentajes de recaudación durante la emergencia sanitaria Covid-19, los diferentes sectores del país tuvieron que acoplarse a una situación que les impedía realizar sus actividades regulares (Moreira, 2018).

La Organización Mundial de la Salud (OMS) declaro emergencia sanitaria Covid-19, el 2019 a nivel mundial, mediante en Ecuador fue declarada en marzo del 2020, extendida a sesenta (60) días dentro el territorio nacional, en donde la suspensión de jornada laboral del sector público y privado que además de suspender el derecho a la libertad de tránsito, asociación y reunión, esto

154 UNESUM-Ciencias. Publicación cuatrimestral. Vol. 6, Año 2022, No. 2 (Especial Agropecuaria) 
es participe a la baja en los valores declaratorios dentro del sector comercial del cantón Pedro Carbo (Bazan, 2021).

Los comerciantes aplicaron las predisposiciones que se implementaron por el Comisión de Operaciones de Emergencia Nacional (COE), los mercantes cedieron al cierre de actividad comercial temporal, donde se vio afectado las ventas e indirectamente los ingresos tributarios percibidos por la actividad económica.

Dentro esta organización que busca bajar el índice de contagios dentro del cantón se vio a la necesidad de aplicar las regulaciones que ayudaran a la reactivación económica del cantón y disminuir así la tasa de contagios, delimitando espacios físicos e igual forma el aforo poblacional en los distintos puntos de adquisición de bienes y servicios.

En el cantón Pedro Carbo las actividades comerciales se vieron afectadas por el bajo rendimiento económico que se generó tras la emergencia sanitaria, el COE cantonal resolvió la aplicación de semaforización, estas disposiciones fueron implantadas afectando a los comerciantes; bajo ingreso económico, descenso en la movilidad de consumidores al cantón.

Las recaudaciones de impuestos dentro del cantón descendieron drásticamente de manera a que se busca verificar los datos, el bajo rendimiento económico comercial puede causar el retraso de la declaración y al pago de la misma, por lo cual puede llevar a asentar sanciones o multas monetarias, el gobierno busca de enfatizar con regulaciones temporales el pago de estos impuestos en la cual se trata de verificar si estas fueron aplicadas a los contribuyentes del cantón.

De acuerdo a esto se busca analizar el impacto que se generó en la declaración de impuestos de los comerciantes del cantón Pedro Carbo, ante las regulaciones tributarias y cómo incidieron en los valores de declaración, ya que mediante la actividad mercantil de los comerciantes se retribuye a la Administración Tributaria.

\section{DESARROLLO}

Este trabajo de investigación cuali-cuantitativo con enfoque no experimental, el cual se alcanzó analizar evidencias teóricas y empíricas utilizando métodos y técnicas de recolección de información y datos, con la guía cualitativa se buscó reconocer características del hecho a investigar.

De la misma manera el enfoque cuantitativo los datos empíricos obtenidos mediante la aplicación del método descriptivo pretende acoger de manera lógica datos de las encuestas realizadas a los comerciantes, estableciendo el reconocimiento de sus patrones, los resultados numéricos y verificar de donde nace la problemática y contrastar las conjeturas de la investigación, se empleará un análisis horizontal la cual calcula las variaciones en los resultados tributarios. El método correlacional ayuda a determinar la relación de las variables de la investigación en donde la emergencia sanitaria Covid-19 influyo en las declaraciones tributarias de los comerciantes analizando los diversos factores ocurridos y verificar el grado de relación de nuestras variables.

Se aplicó el método bibliográfico enmarca las bases teóricas se recurrió a utilizar un método bibliográfico a través revistas científicas, libros, informes de titulación y normativas vigentes aportando a fomentar esta investigación con bases e información legal. Método Inductivo nos 
Ricardo Junior Merchan Holguin, Adela del Jesús Lucio Pillasagua

permite elaborar conclusiones de acuerdo las situaciones o sucesos que se hayan desarrollado dentro de la emergencia sanitaria y su impacto a la declaración de impuestos de los comerciantes para tener una perspectiva más clara de las ventajas, desventajas y la adopción de los protocolos de seguridad sanitaria.

Para esta investigación se aplicará la técnica de observación verificando la introducción de consumidores al cantón en mínima capacidad y máxima capacidad verificando el estado actual de las actividades económicas de los contribuyentes ayudándose con el instrumento de recolección de información teórica "Ficha de observación".

La técnica de encuesta ayudó tomando en cuenta la población del sector comercial, para sistematizar información de comerciantes independientes, para verificar de manera más amplia los acontecimientos que llegaron a suscitarse durante un fenómeno que haya causado esta situación, se aplicó una escala de Likert, verificando de manera cuantitativa los resultados, los mismos que se ejecutó un análisis de Cronbach con la utilización del Software IBM SPSS para ejecutar un aprueba de fiabilidad ya que este sistema nos ayuda con un análisis estadístico avanzado, para así tener una mejor perspectiva analítica de estos datos.

\section{Resultados y discusión}

Mediante el método de la observación sociedades pequeñas y medianas la aplicación de normativas por los COE-cantonales en donde la disminución de aforo disminuye debido a la emergencia sanitaria Covid-19, además de afectar a los transportistas que trasladan a los consumidores al cantón, el cambio de semaforización de rojo a amarillo para reactivar la economía en julio del 2020 en el cantón, disminuir el aforo representa un porcentaje menor en posibles ingresos económicos.

La presente investigación se relaciona entre las declaraciones tributarias de los comerciantes y el impacto que causo la emergencia sanitaria Covid-19 del cantón Pedro Carbo, se da importancia en las declaraciones, recaudaciones y pagos de estos impuestos, dichos valores se recaudados por el Servicio de Rentas Internas periódicamente.

\section{Validación de encuestas - Prueba de fiabilidad}

Dentro de la investigación se ejecutó una prueba de fiabilidad después de la recopilación de información en las encuestas ejecutadas a lo que demuestra lo siguiente:

Tabla 1. Prueba de Fiabilidad de Encuestas.

\section{Resumen de procesamiento de casos}

\begin{tabular}{llc|c}
\hline & $\mathbf{N}$ & $\mathbf{\%}$ \\
\hline Casos & Válido & 238 & 100,0 \\
\cline { 2 - 3 } & Excluido $^{\text {a }}$ & 0 &, 0 \\
\hline Total & 238 & 100,0 \\
\hline
\end{tabular}

Estadísticas de fiabilidad

\begin{tabular}{l|l|l}
\hline Alfa de Cronbach & $\begin{array}{l}\text { Alfa de Cronbach basada } \\
\text { en elementos estandarizados }\end{array}$ & N de elementos \\
\hline $\mathbf{7 1 7}$ &, 616 & 17 \\
\hline
\end{tabular}

156 UNESUM-Ciencias. Publicación cuatrimestral. Vol. 6, Año 2022, No. 2 (Especial Agropecuaria) 
Una vez ejecutada las encuestas a lo que nos arrojó un 0,717 de fiabilidad de las respuestas de nuestras preguntas realizadas con la escala de Likert a los 238 contribuyentes de nuestra muestra de investigación realizada en el Cantón Pedro Carbo, siguiendo con la metodología aplicada.

\section{Análisis de encuestas realizadas a los comerciantes del Cantón Pedro Carbo.}

1. Las declaraciones de impuestos durante la emergencia fueron:

Tabla 2 Las declaraciones de impuestos durante la emergencia.

\begin{tabular}{lcc}
\hline \multicolumn{1}{c}{ Opciones } & Frecuencia & Porcentaje \\
\hline Altas & 10 & $4 \%$ \\
Medianamente altas & 24 & $10 \%$ \\
Normales & 46 & $19 \%$ \\
Bajas & 137 & $58 \%$ \\
Muy bajas & 21 & $9 \%$ \\
\hline TOTAL & $\mathbf{2 3 8}$ & $\mathbf{1 0 0 \%}$ \\
\hline
\end{tabular}

Fuente: Comerciantes del Cantón Pedro Carbo.

Dentro de la tabla 2 los 137 comerciantes del cantón Pedro consideran que las declaraciones tributarias bajaron durante la Emergencia sanitaria Covid-19 que representa un 58\%, de igual manera el 46 de estos consideras que las declaraciones se mantuvieron de forma normal y que 24 de ellos consideraron que fueron medianamente altas a lo que expresa un pequeño incremento en sus declaraciones.

2. ¿Las ventas de su comercial bajaron mediante a la emergencia sanitaria Covid- 19 ?

Tabla 3 Las ventas durante la Emergencia Sanitaria COVID-19.

\begin{tabular}{lcc}
\hline Opciones & Frecuencia & Porcentaje \\
\hline Muy de acuerdo & 52 & $22 \%$ \\
De acuerdo & 141 & $59 \%$ \\
Ni de acuerdo ni en desacuerdo & 25 & $11 \%$ \\
En desacuerdo & 19 & $8 \%$ \\
Totalmente en desacuerdo & 1 & $0 \%$ \\
\hline TOTAL & $\mathbf{2 3 8}$ & $\mathbf{1 0 0 \%}$ \\
\hline
\end{tabular}

Fuente: Comerciantes del Cantón Pedro Carbo.

Por otra parte, en la tabla 3 se pudo demostrar que 238 comerciantes encuestados dentro del Cantón el 59\% están de acuerdo que las ventas bajaron mediante a la emergencia sanitaria, el $22 \%$ están Muy de acuerdo que estas ventas bajaron, $11 \%$ no está de acuerdo ni desacuerdo y el 
Ricardo Junior Merchan Holguin, Adela del Jesús Lucio Pillasagua

8\% de estos están en desacuerdo de que las ventas bajaran en el cantón durante la emergencia sanitaria COVID-19.

3. ¿Existen factores que afectaron la declaración y pago de los tributos durante la emergencia sanitaria?

Tabla 4 Existen Factores que afectan la declaración y pago de tributos.

\begin{tabular}{lll}
\hline Opciones & Frecuencia & Porcentaje \\
\hline Muy frecuentemente & 7 & $3 \%$ \\
Frecuentemente & 24 & $10 \%$ \\
Ocasionalmente & 38 & $16 \%$ \\
Raramente & 88 & $37 \%$ \\
Nunca & 81 & $34 \%$ \\
\hline TOTAL & $\mathbf{2 3 8}$ & $\mathbf{1 0 0 \%}$ \\
\hline
\end{tabular}

Fuente: Comerciantes del Cantón Pedro Carbo.

\section{Análisis e interpretación}

En la tabla 4 de los 238 encuestados en el Cantón Pedro Carbo los comerciantes mencionaron que $37 \%$ de estos presentan Raramente factores que afectan en la declaración y pago de impuestos el $34 \%$ mencionan que Nunca hay Factores que le impiden realizar sus declaraciones y pagos de tributos, $16 \%$ menciona que Ocasionalmente existen estos factores y el 10\% presentan factores al momento de declarar y al pago de estos y el 7\% Muy Frecuentemente los presentan.

4. ¿Cuántos días estuvo sin actividad comercial (cerrado su local) por la emergencia sanitaria Covid-19?

Tabla 5 Sin actividad comercial durante la Emergencia Sanitaria COVID-19.

\begin{tabular}{lcc}
\hline Opciones & Frecuencia & Porcentaje \\
\hline De 1 a 20 días & 18 & $8 \%$ \\
De 20 días a 40 días & 6 & $3 \%$ \\
De 40 días a 60 días & 13 & $5 \%$ \\
De 1 mes a 2 meses & 56 & $24 \%$ \\
De 2 meses a 4 meses & 145 & $61 \%$ \\
\hline TOTAL & $\mathbf{2 3 8}$ & $\mathbf{1 0 0 \%}$
\end{tabular}

Fuente: Comerciantes del Cantón Pedro Carbo.

Se pudo verificar que dentro del Cantón Pedro Carbo los encuestados el $61 \%$ de estos estuvieron de 2 meses a 4 meses sin actividad comercial, de 1 mes a 2 meses sin actividad comercial el 24\%, el $8 \%$ de 1 a 15 días y de 20 días a 30 días un 5\% estuvieron sin actividad comercial.

5. ¿Después de que el COE-M efectuara la reducción de semaforización dentro del cantón las ventas mejoraron?

Tabla 6 Cambio de semaforización.

158 UNESUM-Ciencias. Publicación cuatrimestral. Vol. 6, Año 2022, No. 2 (Especial Agropecuaria) 


\begin{tabular}{lcc}
\hline Opciones & Frecuencia & Porcentaje \\
\hline Muy de acuerdo & 30 & $13 \%$ \\
de acuerdo & 143 & $60 \%$ \\
ni de acuerdo ni desacuerdo & 47 & $20 \%$ \\
en desacuerdo & 18 & $8 \%$ \\
Totalmente en desacuerdo & 0 & $0 \%$ \\
\hline TOTAL & $\mathbf{2 3 8}$ & $\mathbf{1 0 0 \%}$ \\
\hline
\end{tabular}

Fuente: Comerciantes del Cantón Pedro Carbo.

Para los 238 comerciantes del cantón Pedro Carbo mencionaron que están de acuerdo el 60\% después de que el COE-M efectuará la reducción de semaforización dentro del cantón las ventas mejoraron, el $20 \%$ ni de acuerdo ni des acuerdo de que las ventas mejoraran y un 13\% están Muy de acuerdo en que las ventas mejoraron dentro el cantón.

6. ¿Cómo se siente con las medidas del COE-M empleadas durante la emergencia sanitaria?

Tabla 7 Medidas del COE- Municipal.

\begin{tabular}{lcc}
\hline Opciones & Frecuencia & Porcentaje \\
\hline Muy de acuerdo & 14 & $6 \%$ \\
De acuerdo & 132 & $55 \%$ \\
Ni de acuerdo ni en desacuerdo & 51 & $21 \%$ \\
En desacuerdo & 37 & $16 \%$ \\
Totalmente en desacuerdo & 4 & $2 \%$ \\
\hline TOTAL & $\mathbf{2 3 8}$ & $\mathbf{1 0 0 \%}$
\end{tabular}

Fuente: Comerciantes del Cantón Pedro Carbo.

Dentro de los 238 comerciantes encuestados el 55\% de estos menciono que está de acuerdo con las medidas empleadas por el COE-municipal durante la emergencia sanitaria, el $21 \%$ no está ni de acuerdo ni desacuerdo con las medidas empleados por la comisión, el $16 \%$ está en total desacuerdo con estas medidas y el 6\% están Muy de acuerdo con las medidas implementadas por el CEO- municipal o cantonal.

\section{Discusión}

La Emergencia sanitaria declarada en el 2020 en Ecuador trajo consigo muchas inquietudes en el país, de este virus afecto la parte social, económica y salud de los ciudadanos, las autoridades implementaron protocolos de bioseguridad que ayudaron a solventar los contagios que se propagaban en el país como lo explica (Caiza, 2021).

Esta serie de acontecimientos tanto como la emergencia sanitaria y el impacto que tuvo las declaraciones tienen un gran apego a la actividad realizada por los comerciantes debido que el valor de sus ingresos económicos por su actividad debe ser debidamente declaradas en los periodos establecidos por la Administración tributaria como lo describe (Cando, 2021). 
Ricardo Junior Merchan Holguin, Adela del Jesús Lucio Pillasagua

En Ecuador se resolvieron reformas tributarias empleadas durante la emergencia sanitaria Covid19 a las que suspende los plazos y términos de cobro de las Administración tributaria empleadas desde marzo del 2020 hasta el mes de junio del mismo periodo, con la aplicación de estas reformas dirigidas para todos los contribuyentes del país, mencionado por Alava \& Barahona, (2021), el 16 de junio se reanudaron los procesos de la Administración Tributaria salvo al caso de los cantones en los que el COE- Cantonal declara hábil su semaforización.

En comparación de análisis se utilizará los periodos del 2019 y 2020, a lo cual podemos observar un descenso total de ingresos recaudados del $13 \%$ en el 2020 de impuestos directos e indirectos, aunque la Administración Tributaria superara sus metas de recaudaciones tributarias estos no implican el impacto o déficit de ingresos en sus declaraciones durante la emergencia sanitaria Covid-19, ya que mediante su recaudación neta sufrió un descenso del 13\% en sus recaudaciones a lo que representa a 1.653.868.434,00 millones de dólares en comparación a los periodos del 2019 y 2020 a referencia a lo expresado por Caiza, (2021).

Tabla 8 Recaudaciones de los periodos.

\begin{tabular}{lcccc}
\cline { 2 - 5 } & $\mathbf{2 0 1 9}$ & $\mathbf{2 0 2 0}$ & $\begin{array}{c}\text { Valor } \\
\text { Absoluto }\end{array}$ & $\begin{array}{c}\text { Valor } \\
\text { Relativo }\end{array}$ \\
\hline $\begin{array}{l}\text { Recaución neta (considerando valores } \\
\text { ocasionales) }\end{array}$ & $13.180 .846,2$ & $11.526 .977,7$ & $-1653868,43$ & $-0,13$ \\
\hline
\end{tabular}

Este comportamiento se debe a que se suspendió toda actividad en las que se vincule a la población en espacios físicos ya que la enfermedad podría propagarse de manera exponencial, los COE- Cantonales resolvieron aplicar la semaforización en los diversos cantones a lo que expresa (Jumbo, 2021).

Dentro de la emergencia sanitaria Covid -19, los ingresos declarados por los contribuyentes en el país a comparación al periodo preliminar (2019) y su año venidero (2020) en análisis de información del (Servicio de Rentas Internas, 2020), se puede verificar el impacto en déficit de ingresos declarados al SRI, a la cual se puede observar sobre el Impuestos a la Renta de un $8 \%$ de descenso, a la que respecta a las que más déficit de ingresos el Anticipo al Impuesto a la Renta es del $-26 \%$ y en las declaraciones de Impuesto a la Renta en donde desciende a un $12 \%$ de ingresos a la administración tributarias esta declaración se vincula a las Personas Naturales, Personas Jurídicas y a Herencia, legados y donaciones a el cual no sufrió ningún descenso.

A lo que respecta a el Impuesto al Valor Agregado los ingresos de declaración bajaron un 16\% de las ventas a lo que respecta con menos de 791.776,80 dólares, el Impuesto Ambiental Contaminación Vehicular descendió un $94 \%$ de ingresos siendo un relevante antes las declaraciones de impuestos, el Impuesto Redimible Botellas Plásticas no Retornable tuvo un descenso del 19\%, dentro las Regalías, patentes y utilidades de conservación minera consideradas contribuciones pagados en periodos bajo un 40\%, cabe recalcar que la Contribución para la atención integral del cáncer aumento un $8 \%$ estos impuestos representan un $87 \%$ de ingresos al SRI.

Los ingresos declarados por las entidades competentes mediante las conciliaciones de comercialización de bienes, donde se vincula el IVA (Impuesto al Valor Agregado)

160 UNESUM-Ciencias. Publicación cuatrimestral. Vol. 6, Año 2022, No. 2 (Especial Agropecuaria) 
Publicación cuatrimestral. Vol. 6, No. 2 (Especial Agropecuaria), Año 2022. Pág. 153-168 IMPACTO DE LA EMERGENCIA COVID-19 EN LA DECLARACIÓN DE IMPUESTOS

disminuyendo un 22\% e ICE (Impuesto a los Consumos Especiales) con una disminución del $37 \%$, vinculadas a las importaciones descendieron una totalidad del $23 \%$ en el 2020.

Dentro de las recaudaciones por cantones se manifestaron los mismos déficits de ingresos declarados según como lo expresa Andrade \& Cevallos, (2020), en donde explica la cultura tributaria dentro el país y sus variaciones en los sectores comerciales, este déficits lo podemos notar en el siguiente recuadro:

Tabla 10 Ingresos de declarados a la Administración tributaria por cantones.

\begin{tabular}{|c|c|c|c|c|c|}
\hline \multicolumn{6}{|c|}{ Ingresos de declarados a la Administración Tributaria por Provincia } \\
\hline No. & $\begin{array}{l}\text { Provincia } \\
\text { / Cantón }\end{array}$ & $\begin{array}{c}\text { Total } \\
\text { Recaudación } \\
2019 \\
\end{array}$ & $\begin{array}{c}\text { Total } \\
\text { Recaudación } \\
\mathbf{2 0 2 0} \\
\end{array}$ & Valor Absoluto & $\begin{array}{l}\text { Valor } \\
\text { Relativo }\end{array}$ \\
\hline 1 & Azuay & $698.671 .080,90$ & $570.032 .006,96$ & $-128.639 .073,94$ & $-18 \%$ \\
\hline 2 & Bolivar & $12.067 .752,26$ & $10.258 .144,85$ & $-1.809 .607,41$ & $-15 \%$ \\
\hline 3 & Cañar & $38.162 .666,54$ & $36.184 .936,55$ & $-1.977 .729,99$ & $-5 \%$ \\
\hline 4 & Carchi & $20.511 .898,02$ & $17.822 .297,67$ & $-2.689 .600,35$ & $-13 \%$ \\
\hline 5 & Chimborazo & $70.807 .637,98$ & $59.357 .628,56$ & $-11.450 .009,42$ & $-16 \%$ \\
\hline 6 & Cotopaxi & $119.541 .279,70$ & $105.289 .573,27$ & $-14.251 .706,43$ & $-12 \%$ \\
\hline 7 & El oro & $206.758 .376,84$ & $184.912 .623,06$ & $-21.845 .753,78$ & $-11 \%$ \\
\hline 8 & Esmeraldas & $63.208 .302,65$ & $50.368 .496,40$ & $-12.839 .806,25$ & $-20 \%$ \\
\hline 9 & Galápagos & $36.907 .094,21$ & $20.721 .255,37$ & $-16.185 .838,84$ & $-44 \%$ \\
\hline 10 & Guayas & $4.420 .348 .965,57$ & $4.142 .227 .568,95$ & $-278.121 .396,62$ & $-6 \%$ \\
\hline 11 & Imbabura & $111.697 .099,62$ & $97.577 .180,25$ & $-14.119 .919,37$ & $-13 \%$ \\
\hline 12 & Loja & $81.239 .305,63$ & $65.138 .846,67$ & $-16.100 .458,96$ & $-20 \%$ \\
\hline 13 & Los ríos & $81.550 .916,05$ & $82.263 .716,64$ & $712.800,59$ & $1 \%$ \\
\hline 14 & Manabí & $263.009 .976,18$ & $232.180 .814,32$ & $-30.829 .161,86$ & $-12 \%$ \\
\hline 15 & Morona Santiago & $18.123 .869,93$ & $16.088 .071,23$ & $-2.035 .798,70$ & $-11 \%$ \\
\hline 16 & Napo & $12.426 .924,28$ & $9.109 .223,22$ & $-3.317 .701,06$ & $-27 \%$ \\
\hline 17 & Orellana & $33.080 .322,27$ & $29.063 .024,19$ & $-4.017 .298,08$ & $-12 \%$ \\
\hline 18 & Pastaza & $13.591 .103,17$ & $11.597 .068,45$ & $-1.994 .034,72$ & $-15 \%$ \\
\hline 19 & Pichincha & 7.465.893.122,20 & $6.268 .763 .671,07$ & $-1.197 .129 .451,12$ & $-16 \%$ \\
\hline 20 & Santa Elena & $30.653 .220,03$ & $26.528 .603,06$ & $-4.124 .616,97$ & $-13 \%$ \\
\hline 21 & $\begin{array}{l}\text { Santo Domingo de los } \\
\text { Tsachilas }\end{array}$ & $81.349 .124,75$ & $70.238 .120,71$ & $-11.111 .004,04$ & $-14 \%$ \\
\hline
\end{tabular}


Ricardo Junior Merchan Holguin, Adela del Jesús Lucio Pillasagua

\begin{tabular}{llrrrr}
22 & Sin asignar & $1.508 .624,60$ & $891.286,90$ & $-617.337,70$ & $-41 \%$ \\
23 & Sucumbíos & $22.094 .274,03$ & $18.178 .476,45$ & $-3.915 .797,58$ & $-18 \%$ \\
24 & Tungurahua & $254.855 .148,13$ & $193.579 .833,58$ & $-61.275 .314,55$ & $-24 \%$ \\
25 & Zamora Chinchipe & $110.785 .088,32$ & $63.834 .147,92$ & $-46.950 .940,40$ & $-42 \%$ \\
\cline { 2 - 6 } & Total Recaudación & $\mathbf{1 4 . 2 6 8 . 8 4 3 . 1 7 3 , 8 7}$ & $\mathbf{1 2 . 3 8 2 . 2 0 6 . 6 1 6 , 3 1}$ & $\mathbf{- 1 . 8 8 6 . 6 3 6 . 5 5 7 , 5 6}$ & $\mathbf{- 1 3 \%}$ \\
\hline
\end{tabular}



Figura 1 Ingresos de declarados a la Administración tributaria por cantones.

Dentro la tabla se puede verificar que la mayoría de cantones presenta déficit de recaudación, de la cual toma referencia a (Alava \& Barahona, 2021), en las provincias del Ecuador los ingresos tributarios bajaron de manera drástica como la provincia de Pichincha su recaudación bajo un $16 \%$ durante el periodo 2020, la provincia del Guayas con un déficit de $6 \%$ que representarías a 278 millones de dólares en recaudaciones directas eh indirectas, la provincia del Azuay tiene una baja de $18 \%$ de recaudación a consideración del 2019, la provincia de Tungurahua tiene una baja de 24\%, Manabí con un $6 \%$ con 30 millones de dólares menos de recaudación, todas las bajas se comparan con las recaudaciones del periodo 2019, ya que aún no hay datos oficiales de ingresos tributaros del 2021.

\section{Ingresos por pago de declaraciones de impuestos del cantón Pedro Carbo}

Dentro de los ingresos a la Administración Tributaria las declaraciones de los contribuyentes registrados en el catastro del SRI o quien se le atribuyen estas obligaciones como lo expresas (Bazan, 2021), esta información se encuentra en la página oficial Intersri del Servicio de Rentas Internas a lo que se distribuye los contribuyentes del cantón, en donde se enmarca de la siguiente manera:

Tabla 11 Distribución de contribuyentes dentro del Cantón Pedro Carbo.

162 UNESUM-Ciencias. Publicación cuatrimestral. Vol. 6, Año 2022, No. 2 (Especial Agropecuaria) 
Publicación cuatrimestral. Vol. 6, No. 2 (Especial Agropecuaria), Año 2022. Pág. 153-168 IMPACTO DE LA EMERGENCIA COVID-19 EN LA DECLARACIÓN DE IMPUESTOS

\begin{tabular}{lc}
\hline \multicolumn{2}{c}{ Distribución de contribuyentes dentro del Cantón Pedro Carbo } \\
\hline Activos & 1.574 \\
Suspendidos & 3.751 \\
Pasivos & 752 \\
\hline Total & $\mathbf{6 . 0 7 7}$ \\
\hline
\end{tabular}

De estos se conforma según su tipo de contribuyente, como Personas Naturales con 5.126 a los cuales 1.375 se encuentran de manera activo, 3.751 se encuentran suspendidos, 78 suspendieron sus actividades en el periodo del 2020, como Personería Jurídica se encuentran registradas 490 sociedades de las cuales se enmarcan 199 sociedades activas dentro del cantón y 291 se encuentran de manera pasiva que se puede expresar que han cesado de sus actividades mercantiles.

Tabla 12 Ingreso por declaraciones del Cantón Pedro Carbo.

\begin{tabular}{lrrrr}
\hline \multicolumn{5}{c}{ Ingresos de declaraciones del Cantón Pedro Carbo } \\
\hline Meses & $\mathbf{2 0 1 9}$ & $\mathbf{2 0 2 0}$ & Valor Absoluto & Valor relativo \\
\hline Enero & $215.651,01$ & $141.892,30$ & $-73.758,71$ & $-34 \%$ \\
Febrero & $97.163,88$ & $172.552,09$ & $75.388,21$ & $78 \%$ \\
Marzo & $181.128,26$ & $109.859,21$ & $-71.269,05$ & $-39 \%$ \\
Abril & $105.247,32$ & $129.644,58$ & $24.397,26$ & $23 \%$ \\
Mayo & $128.009,39$ & $69.316,13$ & $-58.693,26$ & $-46 \%$ \\
Junio & $87.139,52$ & $90.953,60$ & $3.814,08$ & $4 \%$ \\
Julio & $107.980,28$ & $79.335,71$ & $-28.644,57$ & $-27 \%$ \\
Agosto & $125.320,72$ & $85.395,88$ & $-39.924,84$ & $-32 \%$ \\
Septiembre & $150.031,97$ & $95.176,91$ & $-54.855,06$ & $-37 \%$ \\
Octubre & $98.736,54$ & $80.881,92$ & $-17.854,62$ & $-18 \%$ \\
Noviembre & $102.569,78$ & $91.112,23$ & $-11.457,55$ & $-11 \%$ \\
Diciembre & $103.484,03$ & $132.285,44$ & $28.801,41$ & $28 \%$ \\
\hline \multicolumn{1}{c}{ TOTAL } & $\mathbf{1 . 5 0 2 . 4 6 2 , 7 1}$ & $\mathbf{1 . 2 7 8 . 4 0 6 , 0 0}$ & $\mathbf{- 2 2 4 . 0 5 6 , 7 1}$ & $\mathbf{- 1 5 \%}$ \\
\hline
\end{tabular}

Dentro del cantón en comparación del 2019 y 2020 se registran ingresos de declaraciones tributarias de USD 1.502.462 dólares americanos y USD 1.278.406 dólares, a esto se puede ver este gran impacto que trajo en si la emergencia sanitaria covid-19 como lo expresa (Cevallos, Calle, Ponce, 2020), a lo que podemos denotar en el siguiente recuadro expresado en los ingresos declarados por los contribuyentes del cantón.

Figura 2 Ingresos de declaraciones tributarias del cantón Pedro Carbo. 




De esta se puede verificar durante los meses de los periodos del 2019 y 2020, el impacto negativo durante los ingresos declaratorios durante la emergencia sanitaria Covid-19 en cuanto a los ingresos a la Administración Tributaria durante los meses de enero se una baja anticipada ya que la emergencia sanitaria fue decretada en marzo del 2020, con un 34\% de déficit de comienzo del periodo, en el mes marzo se ve un descenso del 39\%, aunque en abril se ven un aumento del $26 \%$, no cabe negar que para esta fecha ya estaba en vigencia el decreto de la Emergencia Covid19 y en mayo se ven un descenso bastante significativo del 46\% con un valor de USD 58.693,26 dólares, para junio se ve un aumento significativo del $4 \%$ que a sus posteriores meses como es julio, agosto y septiembre los porcentajes bajaron de manera exponencial hasta un $37 \%$, para octubre y noviembre se vio un descenso del 18 y $11 \%$ consecutivamente, a la cual se recupera en diciembre con las festividades, cabe recalcar que para esta fecha ya había bajado la semaforización del COE- Cantonal para reactivar la economía.

Para el cantón Pedro Carbo de los ingresos por declaración tributara a la Administración tributaria en comparación de los periodos antes mencionados se ve una drástica disminución de ingresos de los 12 meses de un periodo los meses de Enero, marzo, mayo, julio, agosto, septiembre, octubre, noviembre del 2020 hay bajas muy importantes esto se debe a el cumplimiento de los decretos presidenciales en donde el cierre de los negocios afectos a la economía del cantón e indirectamente a los montos de declaración tributaria, con un déficit de 15\% menos en comparación al periodo del 2019 en donde aún no se decretaba la emergencia sanitaria.

Para esto el COE- cantonal como comisión encargada resuelve siguiendo las directrices del COE - Nacional y sus directrices como lo expresa (Jumbo, 2021), en donde en las primeras semanas de mayo se adopte la semaforización en rojo dentro del cantón regulando las medidas a aplicar en donde los comerciantes tuvieron que cerrar sus locales de manera temporal, siguiendo las predisposiciones de las órganos regulatorios para disminuir los casos de contagio de la emergencia sanitaria, a la cual el 13 de mayo se resuelve la ampliación de la misma semaforización hasta el 31 de mayo del 2020, cabe recalcar que los comerciantes de productos de

164 UNESUM-Ciencias. Publicación cuatrimestral. Vol. 6, Año 2022, No. 2 (Especial Agropecuaria) 
canasta básica y de productos de agricultura de consumos diario no cesaron su atención, así los moradores puedan hacer uso de estas, adaptándose a las medidas de seguridad sanitaria extendidas por el COE- Cantonal.

Ya para el mes de Junio se implementó los planes pilotos de cambio de semaforización a Amarillo para no entrar a un estancamiento económico dentro del cantón y reactivar a la economía del mismo, pero optando con todas las medidas y restricciones que implementa el comité, aun así varios de los comerciantes mantuvieron cerrados los locales por la poca entrada de consumidores en donde no había cooperativas de transporte interprovincial ni inter cantonal, ya que hay una gran demanda de consumismo que se acerca al cantón, y que además siguiendo con las predisposiciones de categorización de semaforización donde muchos de los locales no pudieron abrir las puertas.

En el Cantón Pedro carbo la economía se deriva en la comercialización de bienes como productos de hogar de todo tipo, productos de línea blanca, productos de canasta básica, servicio técnico de telefonía, ventas de tecnología y accesorios, prendas de vestir, servicios de constructoras, ventas de productos para la construcción, servicios de asesoría legal, económica, venta de productos agrícolas, productos de protección agrícola, entre otras.

Desde el mes de julio del 2020 a los mes octubre, noviembre y enero del año venidero, se mantiene la implementación del mismo plan de contingencia y manteniendo la misma semaforización en Amarillo, aplicando los protocolos sanitarios ampliando las plazas de comercialización y de ventas de muchos de los locales, plazas de comercialización de productos agrícolas y de los demás servicios antes mencionados, que esta dio un gran resultado ya que la economía del Cantón comenzó a recuperarse para el mes de diciembre del 2020 a lo que se espera una buena recuperación en los siguientes meses en el cantón..

Dentro de esta investigación los 238 comerciantes encuestados en el cantón todos registrados mediante RUC, los encuestados expresaron con 58\% de estos consideran que las declaraciones de impuestos fueron bajas en el periodo esto se debe a las ventas bajaran mediante a la emergencia sanitaria covid-19 del cual el 59\% estuvo de acuerdo, aunque las medidas expedidas por el COECantonal son para precautelar la integridad física de sus gobernados sin desestimar sus necesidades, esta medidas afectaron drásticamente a la economía de los comerciantes de la cual el $65 \%$ de estos estuvo de acuerdo.

Dentro de las declaraciones de impuestos los comerciantes manifestaron que contratan a un contador para poder declarar sus impuestos con la mayoría en porcentaje y de igual manera el $25 \%$ de estos manifestó que ellos mismos hacen sus declaraciones, dentro de estas declaraciones se el $16 \%$ de los contribuyentes fueron sancionados por no pagar en las fechas establecidas por la administración tributaria, y que un $37 \%$ de estos tuvieron factores que afectaron el pago de tributos y un 10\% de estos manifiestan que estos factores son frecuentes.

A esto se aplicaron reformas tributarias en el pago de sus tributos a los que el $32 \%$ de los comerciantes no están de acuerdo con las reformas tributarias, a lo cual existieron diferimiento mensual o periódica para los contribuyentes a los que se dificultada el pago de estos a los cuales mencionaron con un 55\% de comerciantes encuestados que no se les atribuyo este definimiento. 
Ricardo Junior Merchan Holguin, Adela del Jesús Lucio Pillasagua

El conocimiento de las reformas tributarias empleadas durante la emergencia sanitaria es un factor clave para que los comerciantes se le atribuye los beneficios o salvoconductos que ayuden a impedir cualquier mal entendido frente a la Administración tributaria a lo cual el $62 \%$ no conocen de la reformas implementadas durante la emergencia sanitaria, de igual manera se indago si los comerciantes conocen sobre los porcentajes de declaración del cantón a lo que el $70 \%$ menciono que no conocen sobre el tema.

Dentro las actividades comerciales del cantón en donde $61 \%$ de los comerciantes encuestados menciono que estuvo entre 2 a 4 meses sin actividad comercial, debido a la regulaciones o resoluciones implantadas por el COE-Nacional y Cantonal y la reducción de semaforización las ventas mejoraron a los cual el $60 \%$ estuvo de acuerdo durante el lapso de julio a diciembre aunque viendo las estadísticas de recaudación solo mejoraron en diciembre, de igual manera mencionaron que el comercio informal influyo en el descenso de las ventas y $16 \%$ de los comerciantes se sienten in conformes con estas regulaciones.

\section{CONCLUSIONES}

Los ingresos por declaraciones de impuestos descendieron de manera drástica en comparación del periodo 2019 al 2020 en el Cantón Pedro Carbo alcanzando su máximo pico en mayo con un $46 \%$ menos de ingresos tributarios, marzo con un 39\% menos y septiembre con un $37 \%$ de déficit en ingresos de declaraciones de manera general al año descendió de un $15 \%$ menos a consideración del año 2019, en donde los comerciantes del cantón estuvieron 3 meses sin actividad económica, dentro de este periodo se pudo verificar la suspensión de 78 contribuyentes del sector, tras enfrentarse a la dura realidad económica que se vive dentro de la emergencia sanitaria Covid-19.

Los protocolos implementados mediante las resoluciones para solventar los contagios dentro el cantón afecto indirectamente a las actividades comerciales del cantón disminución de entrada de la demanda de consumidores, bajas ventas, locales sin poder abrir ya que no cumplían con las categorías establecidas, restricciones sanitarias, baja en el aforo dentro de los comerciales, ante lo mencionado afectos ingresos económicos de los comerciantes a lo que hizo que las declaraciones tributarias descendieran de manera drástica.

La declaración de impuestos de los sujetos pasivos descendió un total del 13\% en el 2020 de impuestos a lo que representa a 1.653.868.434,00 millones de dólares en comparación a los periodos del 2019 y 2020, cual se puede observar sobre el Impuestos a la Renta de un 8\% de descenso, el Impuesto al Valor Agregado los ingresos de declaración bajaron un 16\% de las ventas a lo que respecta con menos de 791.776,80 dólares denotando el impacto de ser suscito durante la emergencia sanitaria.

Se verifico el impacto de los ingresos por declaraciones tributarias en los diferentes cantones en la recaudación de impuestos en Ecuador en donde se denoto un descenso del $44 \%$ menos de ingresos en la provincia de Galápagos y del $42 \%$ en Zamora Chinchipe, en la provincia de Pichincha se reflejó un descenso del 16\% que representa a USD 1.197.129.451 millones de dólares, así mismo la provincia del Guayas con USD 278.121.396 millones de dólares, sin dejar de lado las demás provincias que muestran un impacto de déficit en las recaudaciones, a diferencia de la provincia de Los Ríos que ascendió un $1 \%$.

166 UNESUM-Ciencias. Publicación cuatrimestral. Vol. 6, Año 2022, No. 2 (Especial Agropecuaria) 


\section{REFERENCIAS BIBLIOGRÁFICAS}

Alava Bareto, J., \& Barahona Gracia, S. (2021). Impacto Tributario del COVID-19 en Ecuador: Análisis y Estadísticas del Impuesto al Valor Agregado 2019-2020. Revista Ciencia UNESMI ISSN 1390-4272, 87-95. doi:doi:https://doi.org/10.29076/issn.2528-7737vol14iss36.2021pp87-95p

Andrade Donoso, M., \& Cevallos Caza, K. (2020). Cultura tributaria en el Ecuador y sostenibilidad fiscal. e-ISSN: 2697-3413. 6. doi:https://doi.org/10.35290/re.v1n1.2020.290

Bazan, J. (2021). Comportamiento del impuesto al valor agregado en la recaudación a nivel nacional y su incidencia por efecto covid-19 en la provincia de Santa Elena. Pdf, Universidad Estatal Peninsular de Santa Elena. Recuperado el 26 de Noviembre de 2021, de https://repositorio.upse.edu.ec/bitstream/46000/6284/1/UPSE-TCA-2021-0137.pdf

Caiza Clavijo, E. (2021). El impacto del COVID-19 en la recaudación del impuesto al valor agregado IVA, en el sector textil del Ecuador período 2019-2020. Universidad Central del Ecuador, Carrera de Finanzas, Quito. Recuperado el 26 de Noviembre de 2021, de http://www.dspace.uce.edu.ec/bitstream/25000/24813/1/FCECF-CAIZA\%20ERIKA.pdf

Cando Muso, E. (2021). "La recaudación tributaria y el cumplimiento voluntario durante la emergencia sanitaria en la provincia de Tungurahua. Pdf, Universidad Tecnica de Ambato, Ambato. Recuperado el 26 de Noviembre de 2021, de http://repositorio.uta.edu.ec/bitstream/123456789/33928/1/T5165i.pdf

Cevallos Ponce, G., Calle Lino, A., Ponce Cedeño, o., \& O. (2020). Impacto social causado por la covid-19 en ecuador. Investigacion y Pensamiento ISSN:2254-3376. Obtenido de http://repositorio.uotavalo.edu.ec/bitstream/52000/318/1/UO-PG-COM-2021-09.pdf

Echeverria Urgiles, V. (2018). Análisis comparativo de los procesos de gestión del Comité de Operaciones de Emergencia $y$ del Sistema Integrado de Seguridad ECU 9-1-1. Obtenido de https://repositorio.uasb.edu.ec/bitstream/10644/7021/1/T3026-MGRD-Echeverria-Analisis.pdf

Fernandez, G. A. (2020). Comercio informal en ciudades intermedias del Ecuador: Efectos socioeconómicos y tributarios. Revista de Ciencias Sociales, ISSN: 1315-9518 XXVI . Obtenido de https://www.redalyc.org/journal/280/28063519016/28063519016.pdf

Jumbo, B. (12 de enero de 2021). El comercio. Estas son las restricciones que se aplican en un cantón en semáforo rojo. Obtenido de https://www.elcomercio.com/actualidad/ecuador/ecuador-restricciones-semaforo-rojocanton.html

Moreira Loor, H. (2018). La cultura tributaria y su incidencia en el cumplimiento de las obligaciones tributarias de los comerciantes de la Sociedad Abastecedora del Mercado Central de Jipijapa. Universidad Estatal del Sur de Manabí, Facultad de Ciencias Económicas, Jipijapa. Obtenido de http://repositorio.unesum.edu.ec/bitstream/53000/1513/1/UNESUM-ECUADOR-AUDI-2018-62.pdf

Servicio de Rentas Internas. (27 de Noviembre de 2020). InterSRI. Obtenido de https://www.sri.gob.ec/informaciongeneral. 
